\title{
La cavitation dans les pompes
}

\author{
pAR P. GUITON, \\ RAPPORTEUR DU GROUPE DE TRAVAII NUMŔR 2 \\ DE LA COMMISSION POUR L'ETUDE DE LA CAVTTATION DANS LES TURBOMACHINES \\ (Texte résumé, le texte intégral devant être publié ultérieurement)
}

\section{INTRODUCTION}

\section{a) But.}

A la demande de la Commission, le rapport fait le point actuel des connaissances que l'on peut considérer comme acquises concernant la cavitation dans les pompes et remplit les buts suivants :

$1^{\circ}$ Il rassemble, à l'usage des constructeurs et des utilisateurs de turbines, l'essentiel des renseignements sur cette question, afin de leur permettre d'en tirer des remarques utiles pour leurs propres problèmes de cavitation.

$2^{\circ}$ Il constitue l'outil nécessaire aux travaux ultérieurs du groupe sur la cavitation dans les pompes et pour cela :

a) Grée un cadre de chapitres et de paragraphes où toute question ultérieure et tout renseignement viennent trouver leur place:

b) Précise le langage commun de façon que les définitions et les notations adoptées évitent les confusions ultérieures, notamment en ce qui concerne les différents stades de cavitation; c) Etablit un diagramme général de représentation permettant la confrontation des résultats.

3" Il présente à l'aide de ce diagramme quelques résultats types déduits d'une première statistique et les compare avec les formules et abaques classiques.

\section{b) Composition du groupe de travail.}

Le groupe de travail $n^{\circ} 2$ comprend actuellement les personnes suivantes :

M. Gurron (Société Bergeron), rapporteur. MM.

Bouchon (Société Rateau);

Huguenin (Laboratoire d'Hydranlique et de Cavitation de l'ENIAM, Paris);

Manon (Société SW);

DE SAINT-VAuldry (Société Sulzer);

Sasia (Société Worthington-France);

Tenot (professeur).

\section{GÉNËRALITÉS ET NOTATIONS}

\subsection{Généralités.}

\subsubsection{Comparaison ayeg les turbines.}

En rappelant les données générales du problème, ce paragraphe met en relief les principales différences avec les turbines:

- Tension de vapeur souvent beaucoup plus élevée (eau chande, hydrocarbures, etc.);

- Cavitation a l'entrée de la roue et non à la sortie, et par suite influençant beaucoup plus les caractéristiques de fonctionnement;

- Reméde de l'entrée d'air, par suite, beaucoup moins utilisable;

- Bruit parfois inéluctable, et ne pouvant être considéré comme symptomatique d'une cavitation préjudiciable;

- Dépression du liquide souvent bien avant la machine, avec possibilité de début de dégazage favorisant la caritation.
I.1.2. Caymtation phystoue et Cavitation industrielle; STADES DE CaVitation :

- Cavitation physique dès qu'il y a des bulles de cavitation dans la machine. On ne dit pas pour autant, industriellement, que la pompe cavite;

- Cavitation industrielle quand la cavitation altère son fonctionnement (voir II.2.2.).

\section{I.2. Notations.}

Les paramètres intervenant dans l'étude de la cavitation peuvent être répartis en deux catégories :

Première catégorie : Paramètres externes : ceux qui ont trait au comportement extérieur de la machine et auxquels on est limité le plus souvent en l'absence de renseignements sur son dimensionnement interne. 
Deuxizime catégorie: Paramètres internes: ceux faisant intervenir le dimensionnement interne et en particulier le diamètre $D_{a}$ de l'œillard de la $1^{\text {re }}$ roue.

Les premiers intéressent surtout le projeteur et l'utiiisateur ct les seconds le constructeur.

Principaux paramitres externes :

- (NPSH) : Charge nette absolue à l'aspiration: c'est le niveau énergétique du liquide à l'entrée de la pompe, mesuré au-dessus d'un point déterminé de la première roue.

Cette notation a prévalu, provisoirement pent-être, parmi plusieurs autres, comme étant la plus répandue chez les constructeurs et utilisateurs de pompes;

$-n_{s}=\frac{n Q^{1 / 2}}{\mathrm{H}^{3 / 2}}$ : Vitesse spécifique, que l’on propose de remplacer par un nombre sans dimensions :

$$
\omega_{s}=\frac{\omega Q^{3 / 2}}{g^{3 / 2} \mathrm{H}^{2 / 4}}
$$

que l'on pourrait appeler «rapidité spécifique»;

$-\mathrm{S}=\frac{n \mathrm{Q}^{1 / 2}}{(\mathrm{NPSH}) \%} \quad$ Vitesse spécifique d'aspiration, à jemplacer par :

$$
\omega \cdot a=\frac{\omega Q^{1 / 6}}{g^{3 / 2}(\mathrm{NPSH})^{3 / 4}}
$$

rapidilé spécifique d'aspiration.

Principaux parametres internes:

$$
\eta_{a}=\frac{(\mathrm{NPSH})}{\mathrm{U}_{a^{2}} / 2 g} \quad \rho_{a}=\frac{V_{a}}{\mathrm{U}_{a}} \quad \Omega_{a}=\frac{\varphi_{a}^{1 / 2}}{\psi_{a}^{3 / 4}} \quad m=\frac{s_{a}}{\pi \mathrm{D}^{2} / \mathrm{H}}
$$

$\mathrm{U}_{a}$ étant la vitesse périphérique de l'œillard de la $1^{\text {re }}$ roue; $\mathrm{V}_{a}$ la vitesse moyenne dans cet oillard. $\mathrm{V}_{a}=(\mathrm{Q} / \mathrm{sa})$; $s_{a}$ la section utile de l'œillard.

\subsection{Formulaire.}

Les principales relations du rapport $y$ sont rassemblées. A noter que :

$$
\omega_{s}=0,019_{n_{s}} \quad(1) s a=0,019 \mathrm{~S} \quad \Omega_{a}=\frac{\eta \mid m_{n}^{3 / 4}}{\varphi_{a} a^{3 / 4}}=\frac{(1)_{s a} m^{1 / 2}}{\left(8 \pi^{3}\right)^{3 / 4}}
$$

\section{INFLUENCE DE LA CHARGE NETTE ABSOLUE A L'ASPIRATION (NPSH) SUR LES CARACTÉRISTIQUES DE FONCTIONNEMENT DES POMPES}

\section{I1.1. Charge nette absolue à l'aspiration (NPSH).}

La définition de cette grandeur est précisée dans le cas le plus général :

$$
(\mathrm{NPSH})_{\mathrm{d} \text { t } \mathrm{p}}=\frac{\mathrm{P}_{0}}{\gamma_{0}}+\frac{\mathrm{V}_{0}{ }^{2}}{2 g}-\frac{\mathrm{P}_{v}}{\gamma_{0}}
$$

$\mathrm{P}_{0}, \mathrm{~V}_{0}, \gamma_{0}, \mathrm{P}_{\mathrm{v}}$ étant respectivement la pression, la vitesse moyenne, le poids spécifique et la tension de vapeur du liquide à l'entrée de la machine, la pression étant ramenée au niveau de référence.

L'indication disp indique qu'il s'agit du (NPSH) disponible, c'est-à-dire de celui existant effectivement a l'entrée de la machine, lors du fonctionnement considéré et non pas de celui nécessaire pour ne pas caviter : (NPSH) requis. Les (NPSH) correspondant aux divers stades de cavitation sont notés par les indices indiqués en II.5. correspondant à ces stades $(g, c, d, b)$.

Le (NPSH) peut être mesuré, mais il est le plus souvent déduit des données de l'installation. On précise comment il convient de le faire dans le cas le plus général.

II.2. Influence d'une baisse de (NPSH) ${ }_{\text {disp }}$ sur les caractéristiques de fonctionnement d'une pompe.

II.2.1. Counbes $\quad \mathrm{H}=f(\mathrm{NPSH})_{\mathrm{a} 1 \mathrm{sp}}, \quad \mathrm{W}=f(\mathrm{NPSH})_{\mathrm{alsp}} \quad$ ET $\eta=f(\mathrm{NPSH})_{\text {as a a }} n$ ET $Q$ constants.

Des courbes sont données pour diverses vitesses spécifiques, allant des centrifuges lentes aux pompes hélices.

Pour les premières, dès que l'on atteint un certain $(\mathrm{NPSH})_{g}$, il y a chute à pic des courbes caractéristiques (cavitation globale à laquelle correspond l'indice g).
Au fur et à mesure que la vitesse spécifique augmente, cette chute verticale est précédé d'une courbe de raccordement arrondie de plus en plus étendue.

Poul les grandes vitesses spécifiques, cette courbe de raccordement s'étend à tous les débits et pour les pompes hélices fait disparaître même la cavitation globale.

\section{II.2.2. Cavitation industimelze.}

Par définition on appelle cavitation industrielle celle qui correspond pour un débit domné à une chute de hauteur de $3 \%$.

C'est à cette cavitation que correspond l'indice $c$.

\subsubsection{Explicatron schématioue de l'alinure des courbes DE II.2.1.}

On montre que e'est la longueur relative des canaux de la roue qui semble être l'élément déterminant pour l'allure des courbes précédentes. Dans l'ensemble, cette longueur relative diminue avec la vitesse spécifique.

11.3. Modification des courbes caractéristiques de fonctionnement des pompes provoquées par la cavitation.

$\mathrm{Si}$ au lieu de faire décroître (NPSH) dusp à débit constant et vitesse constante, on fait varier le débit à (NPSH) arsp constant et vitesse constante, on obtient les courbes classiques dont l'allure évolue aussi avec la vitesse spécifique.

\subsection{Influence du rognage d'une roue.}

La cavitation dans la roue dépend avant tout des conditions à l'entrée de celle-ci et, à un degré beaucoup moindre, des conditions à la sortie. 
On le montre en diminuant progressivement par rognage le diamètre de sortie d'une même roue.

La cavitation globale se fait toujours pour le mème débit, l'arrondi de raccordement augmentant cependant de plus en plus.

\section{II.5. Caractéristiques de cavitation de l'œillard d'une roue.}

Ces caractéristiques sont données par les courbes $(\mathrm{NPSH})=f(Q)$ à vitesse constante.
On trace, pour autant qu'elles existent, les courbes suivantes:

(NPSH), Cavitation globale;

(NPSH). Cavitation industrielle;

(NPSH) ${ }_{d}$ Point de raccordement;

$(\mathrm{NPSH})_{b}$ Apparition des premières bulles.

La première n'existe pas pour les grands $n_{s}$.

La dernière est le plus sonvent cachée, en l'absence de dispositils de visualisation.

Des coupbes types sont données pour les différents $n_{\star}$.

\section{SIMILITUDE DE CAVITATION DANS LES POMPES ET ÉCARTS PAR RAPPORT A LA SIMILITUDE}

\section{III.1. Similitude de cavitation.}

\section{III.1.1. Similitude aU SEurl de ra cavitation - Courbes $\psi_{a b}=f\left(p_{a}\right)$.}

La différence de pression entre l'entrée de la machine et le point où va apparaître la première bulle suit la lo générale de similitude des turbomachines. Par suite (NPSH) 0 et $\mathrm{H}$ varient comme $\mathrm{U}_{n}{ }^{2} / 2 g$ et restent proportionnels entre eux.

Si l'on ignore $\mathrm{U}_{a}$, on peut seulement faire intervenir leur rapport $\left.\left.\{\text { [(NPSH) })_{b} / \mathrm{H}\right]=\sigma a\right\}$ coefficient de Thoma.

Mais si l'on rogne la roue, $\mathrm{H}$ et par suite ob varient.

Pour les constructeurs, il est done préférable d'écrire séparément :

$$
\begin{aligned}
& \text { pour l'willard } \frac{(\mathrm{NPSH})_{b}}{\mathrm{U}^{2} / 2 g}=\mathrm{Cte}=y_{a b} \\
& \text { et pour la sortie } \frac{\mathrm{H}}{\mathrm{U}^{2} / 2 g}=\mathrm{v}
\end{aligned}
$$

(U étant la vitesse périphérique de sortie).

$c_{a}=\left(V_{a} / U_{a}\right)$ permet le repérage des points de fonctionnement homologues dans l'œillard.

Cette similitude suppose qu'en première approximation la première bulle apparait juste au moment où la pression atteint la tension de vapeur (voir MII.2.1.).

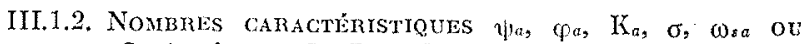
$S$ et $\omega^{\prime}$ sa ou S'. Degré de Cavitation.

Ce paragraphe explicite ce qui a déjà été dit en 1.2 à propos des notations.

- $\omega^{\prime}{ }^{\prime}$ sa et $\mathrm{S}^{\prime}$ diffèrent de $\boldsymbol{o}_{\text {sin }}$ et $\mathrm{S}$ en ce qu'ils font intervenir l'obstruction de l'œillard par le moyeu de la roue;

- Les différents stades de cavitation sont caractérisés par leurs indices $b, d, c, g$, apposés à $\vartheta \mid a, p a, K_{a}, \sigma$, etc.;

- Le degré de cavitation est $a_{c}{ }^{0}=\frac{\mathrm{H}_{0}-\mathrm{H}}{\mathrm{H}_{0}}$

$H_{0}$ étant, pour un débit et une vitesse donnée, la hauteur sans cavitation et $\mathrm{H}$ celle en cavitation.

- La cavitation industrielle est definie par $d_{c}=0,03$.

Comparaison entre $\eta_{a}$ et $\sigma$.

j’a intéresse surtout le constructeur;

$\sigma$ intéresse l'utilisatenr, l'installateur et le projeteur.

Il est précisé que c'est $H_{0}$ et non $H_{\text {quj }}$ doit intervenir au dénominateur de $\sigma$.
Relations entre $\psi_{a}, \varphi_{a}, \mathrm{I}_{a}, \omega_{s a}$ et $\omega^{\prime}{ }_{s a}$ :

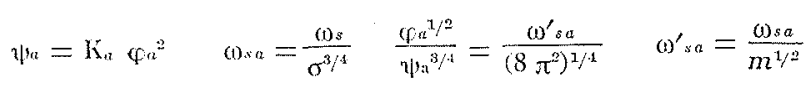

\section{III.2. Similitude en cavitation.}

III.2.1. CONSMDÉRATTONS GRERERTLES.

Il peut y avoir retard ou non à lapparition de la première bulle, suivant que le liquide ne contient pas de gaz dissous, en contient, ou contient même de fines bulles gazeuses formant noyau pour la cavitation, suivant aussi que la durée de passage dans la zone fortement déprimée est plus ou moins longue.

Ouand la cavitation s'est fortement développée, les écarts par rapport à la similitude générale des turbomachines vont en s'acentuant.

Des conditions supplementaires et contradictoires sont à respecter pour passer d'une machine modèle à la machine grandeur (similitule de Froude, identité des temps de passage, similitude de Reynolds).

Elles sont par suite controversées.

Une tendance répandue chez les constructeurs de pompes est de réaliser autant que possible la même hauteur $\mathrm{H}$ pour le modèle et la machine grandeur.

IIT.2.2. Counbes $\psi_{a} a=f\left(\varphi_{a}\right)$ ET $\psi_{a g}=f\left(\varphi_{a}\right)$.

On suppose en première approximation que la similitude générale reste valable, ce qui permet de tracer ces courbes, quitte à examiner séparément les écarts constatés par rapport à la similitude.

\section{III.2.3. Formule de Tenot.}

Formule proposée par le professeur Tenot pour le passage d'une vitesse de rotation à une autre, pour une même machine de petite dimension.

\section{III.3. Ecarts par rapport à la similitude générale.}

III.3.1. ECARTS DUS AU MODE DE RËALISATION DES ESSAIS.

\section{IIr.3.1.1. Présence de gaz dissouls - présence de noyaux} gazeux.

III.3.1.2. Mode de réalisation du (NPSH) a1sp :

a) Variation de la perte de charge à l'aspiration $J_{\Delta}$;

b) Variation de la hauteur géométrique d'aspiration $\mathrm{H}_{A}$;

c) Variation de la pression totale de vapeur et de gaz $P_{A}^{\prime}$ régnant dans un réservoir fermé;

d) Combinaison de $b$ et $c$. Semble la méthode la meilleure. 
III.3.1.3. Indétermination de la courbe en charge.

Il faut être sûr d'avoir au départ une charge suffisante à l'aspiration pour avoir effectivement $\mathrm{H}_{0}$ à tous les débits considérés.

III.3.2. TEMPS DE PASSAGE.

Il semble avoir un rôle important.
III.3.3. Essais D'UNe hême pompe a des vitesses de RotaTION DIFFÉRENTES.

On pense que cac diminue avec la vitesse, mais ce n'est pas sûr, les expériences n’étant pas toutes concordantes.

III.3.4. ESSAIS DE POMPES SEMBLABLES DE DMMENSIONS DIFFERENTES.

En plus de la variation des nombres de Reynolds, de Froude et des temps de passage, il pent $y$ avoir :

- Variation de la rugosité relative;

- Ecarts mineurs dans la similitude géométrique.

CHAPITRE IV

\section{DIAGRAMMES GÉNÉRAUX - FORMULES APPROXIMATIVES}

IV.1. Diagramme général $\psi ; a=f(\psi ; a)$. On trouvera ce dia gramme ci-joint.

IV.1.1. Présentation du Diagramme.

- Coordonnées logarithmiques;

- Courbes $\psi_{a c}=f\left(\varphi_{a}\right)$ des diverses pompes à comparer aux courbes $\omega_{s a}=$ constante et $K_{a}=$ constante;

- Une statistique a été faite parmi les constructeurs. Elle a permis d'établir des courbes types moyennes qui n'ont cependant que valeur d'illustration.

La valeur de la rapidité spécifique $\omega_{s}$ est indiquée sur chaque courbe.

IV.1.2. Principales zones du diagramie $\psi_{a}=f\left(\varphi_{a}\right)$ Courbes Types $\psi_{a c}=f\left(\varphi_{a}\right)$.

On peut constater l'existence de trois zones :

Zone I - Tracés normalux : 0,2 env. $<\varphi_{a}<0,5$ environ;

ZoNe II - Pompes d'extraction: $\varphi_{a}$ petit (grands œillards);

ZoNe III - Certaines pompes pour usages spéciaulx (liquides chargés, etc.) : फa grand (petits cillards).

Le fait que $\varphi$ a varie peu avec la vitesse spécifique pour les roues de tracé normal, à leur point de rendement maximal, conduit à dire : puisque $D_{a}$ varie comme $\sqrt[3]{\text { qa }}$, - Pour un débit donné et une vitesse de rotation donnée, toutes les pompes monocellulaires ont à peu près le mêtme diamètre d'ceillard.

IV.2. Valeurs moyennes de $\omega_{s a}^{\prime}$ (ou $\left.S^{\prime}\right)$ - Courbes $\left.\sigma=f(\omega) s\right)$.

En gros, pour un projet, si l'on veut avoir une marge suffisante, il faut se mettre au-dessus d'une certaine valeur $A$ de $\bar{\omega}^{\prime} s a$.

$$
\text { Par suite : } \sigma=(1 / \mathrm{A})^{1 / 8} \omega s^{4 / 3}
$$

relation à partir de laquelle ont été établies divers abaques, dont ceux de l' «Hydraulic Institute» américain, avec $S=160$ pour les pompes hélico-centrifuges et hélices et $S$ variable de 128 à 180 pour les centrifuges monocellulaires.

Dans un projet, en première approximation, prendre $\mathrm{S} \leqslant 170$ environ pour les tracés normaux sans moyeu dans l'œillard, $S \leqslant 140$ a 150 environ pour les centrifuges avec moyeu dans l'œillard et $S \leqslant 260$ pour les roues d'extraction.

\section{IV.3. Formules de la forme $\psi_{a c}=\mathrm{K}_{1} c_{a} a^{2}+K_{2}$.}

Certains auteurs estiment que l'on peut écrire :

$$
(\mathrm{NPSH})_{c}=\mathrm{K} \frac{\mathrm{V}_{a^{2}}}{2 g}+\lambda \frac{\omega a^{2}}{2 g} \quad(\mathrm{~K} \geqslant 1)
$$

ce qui peut se mettre, en l'absence de prérotation, sous la forme:

$$
\mathrm{y}_{\mathrm{f} a c}=\mathrm{I}_{1} \varphi^{2} \mathrm{a}^{2}+\mathrm{K}_{2}
$$

Diverses valeurs ont été proposées pour $\mathrm{K}$ et $\lambda$, d'où $K_{1}$ et $K_{3}$. Malheureusement aucune ne donne vraiment satisfaction.

\section{IV.4. Relation entre $\omega_{s a}^{\prime}$ et $\omega_{s}$ c'est-à-dire entre $S^{\prime}$ et $n_{s}$ au point de rendement maximal.}

La statistique recueillie ne permet pas de déduire une relation générale. Elle existe cependant pour un constructeur donné dans la mesure où il y a continuité dans ses tracés pour les différentes rapidités spécifiques.

\section{CHAPITRE V}

\section{INFLUENCE DE LA GÉOMÉTRIE DE LA ROUE}

\section{V.I. Influence du diamètre d'œillard $D_{a}$.}

C'est l'influence de $\varphi$.

\section{V.2. Influence de l'épaisseur des aubes à l'entrée.}

Elles créent une obstruction qui, augmentant les vitesses, diminue la capacité d'aspiration pour un débit donné.

\section{V.3. Influence de l'angle d'entrée $D_{1}$.}

Reste du domaine propre de chaque constructeur.

\footnotetext{
V.4. Influence du tracé des aubes proprement dit et du profil de l'arête d'entrée.
}

Reste du domaine propre de chaque constructeur. 


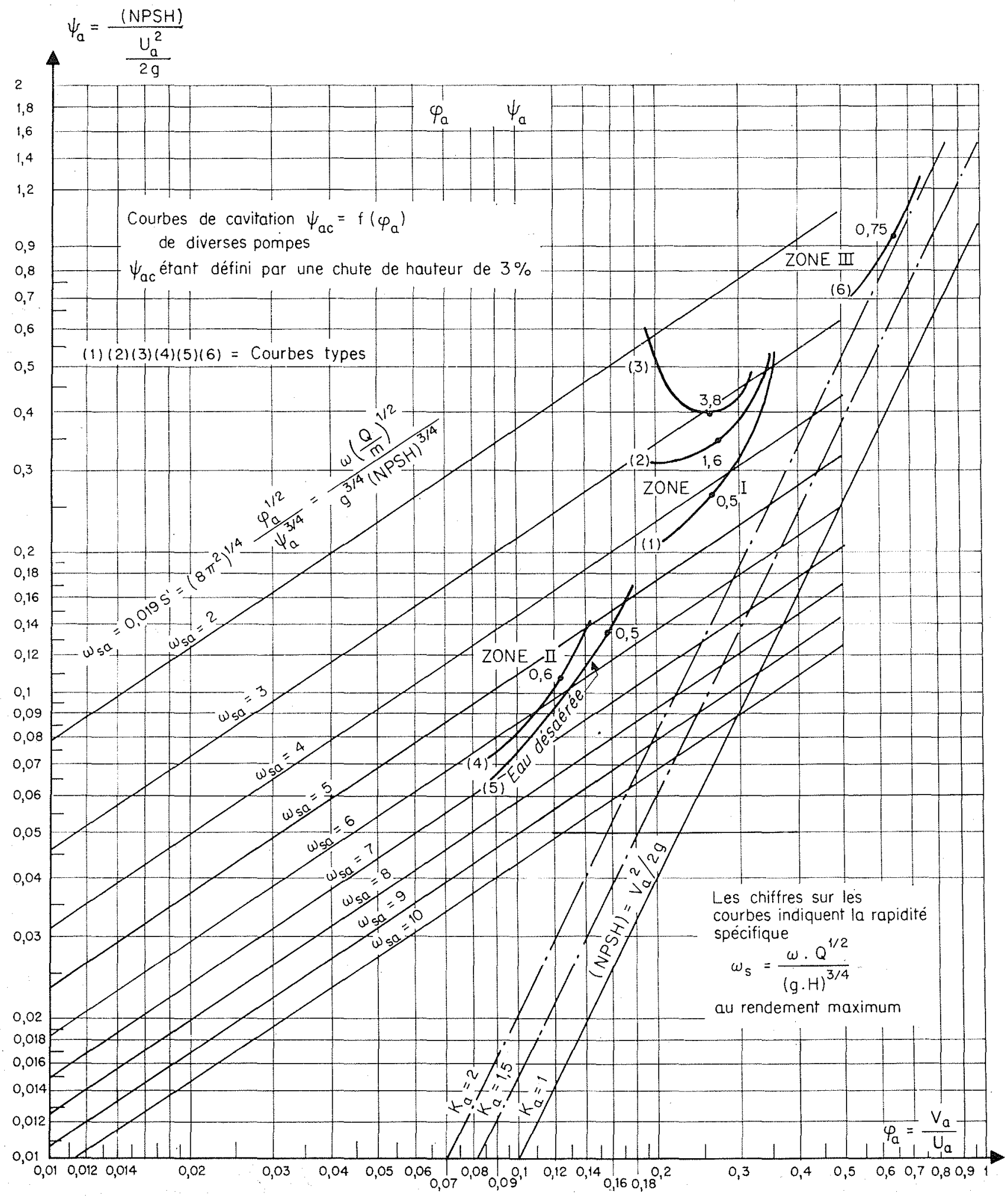

Fig. 1. - Diagramme général de cavitation des pompes. 


\section{V.5. Iufluence du nombre d'aubes.}

Pour les petits $\omega_{x}$, l'augmentation du nombre d'aubes diminue la capacité d'aspiration par suite de l'accroissement de l'obstruction de l'oillard.
Pour les grands $\omega_{x}$, cet effet est contrebalancé par la diminution du couple demandé à chaque pale et par suite, par la diminution de la différence de pression entre intrados et extrados. Il peut y avoir un nombre optimal de pales relativement réduit.

\section{CHAPITRE VI}

\section{CAVITATIONS AUTRES QUE CES CAVITATIONS DANS L'GILLARD DE LA PREMIẼRE ROUE, DANS LA ZONE DE BON RENDEMENT}

\section{VI.1. Cavitation physique à faible débit à l'entrée de la première roue et en amont de celle-ci.}

Au-dessous d'un certain débit, il y a déséquilibre de l'écoulement dans le plan méridien, et apparition d'une nouvelle branche de la courbe $\psi_{a b}=f\left(\varphi_{a}\right)$.

VI.2. Cavitation marginale des roues hélices et des rouets ouverts.

A rapprocher de la cavitation marginale des turbines hélices et Iraplan.

VI.3. Cavitation dans les diffuseurs et les volutes.

VI.3.1. Cavitation Dans tes Diffuseuns,

Elle se traduit par une brusque remontée de la courbe $(\mathrm{NPSH})_{g}=f(\mathrm{Q})$
VI.3.2. Caviration aux becs de volute.

Elle présente une analogie avec celle des diffuseurs, mais affecte rarement les courbes caractéristiques.

VI.4. Cavitation propre aux pompes multicellulaires.

Si la pompe a $m$ roues et si la caractéristique hauteurdébit de $m$ - $t$ roues passe au-dessus de la caractéristique hauteur-débit que l'installation impose à la pompe, les $t$ premières roues et leurs diffuseurs peuvent être en cavitation globale complète, e'est-à-dire fonctionnant à hauteur nulle, en pleine vaporisation pour le débit donné. Cela peut entrainer des grippages des douilles entre étage et bagues d'étanchéité.

\section{ASPECTS PHYSIQUES ET CONSÉQUENCES MÉCANIQUES DE LA CAVITATION}

VII.1. Formes des cavités.

Daus l'ensemble, elles rentrent dans la classification générale établie par le Groupe de travail $n^{\circ} 3$ de la Commission de Cavitation.

\section{VII.2. Bruits ef trépidations.}

On est souvent amené à les accepter pour les pompes quand ils n'entrainent pas de perturbations gênantes du fonctionnement ni d'érosion rapide.

C'est souvent le cas quand ils sont dus à l'écrasement des bulles au sein du liquide.

Une petíte rentrée d'air peut atténuer le bruit sans perturber beaucoup le fonctionmement.

La cavitation globale accentuée est souvent silencieuse.

\section{VII.3. Détériorations.}

VII.3.1. ERosions.

On donne une localisation empruntée au livre de M. Stepanoff sur les principales zones d'érosion dans les pompes.

La cavitation n'entraîne pas d'érosion si l'écrasement des bulles se fait sous faible pression ou si, comme dit plus haut, elle se fait au sein de l'écoulement.

VII.3.2. Disjongtions ET RUPTURES.

Quand il y a fermeture brutale de grosses poches, il peut $y$ avoir déformations ou même ruptures des parois de la roue. Des exemples en sont donnés.

\section{CHAPITRE VIII}

\section{POMPAGE DES LIQUIDES A TENSION DE VAPEUR ÉLEVÉE}

VIII.l. Cas d'un liquide pur : Exemple de l'eau chaude.

L'apparition de la cavitation en son début se fait dans les mêmes conditions que pour l'eau froide désaérée.

Mais dès que le volume de la phase gazeuse n'est ylus négligeable, la masse de vapeur qu'elle contient ne l'est plus non plus. Pour la vaporiser, il a fallu prélever de la chaleur sur l'enthalpie du liquide avoisinant, dont la température s'abaisse légèrement, ce qui correspond à une augmentation apparente du (NPSH) disponible. 
Il $\mathrm{y}$ a alor's freinage à la cavitation.

L'aspect thermodynamique du phénomène est complexe, et continue à laire l'objet de recherches théoriques et expérimentales de la part des constructeurs et spécialistes.

L'écrasement des bulles est moins brutal qu'en eau froide, la vapeur qu'elles contiennent formant matelas élastique.

\section{Cortène de Stepanofr.}

Un critère 13 tenant compte des caractéristiques thermodynamiques du fluide a été introduit par Stepanofl, mais est controversé.

\section{CritĖne de Gage.}

Indique la majoration a donner au (NPSH) nécessaire pour tenir comple des régimes transitoires dans une installation où une pompe à eau chaude aspire par une tuyauterie dans un ballon, compte tenu de leurs volumes respectifs.

\section{VIII.2. Cas d'un mélange : Exemple des hydrocarbuxes.}

S'ils sont composés de liquides purs, leur tension de rapeur suit la loi de Raoult. S'il s'agit d'hydrocarbures complexes, elle peut s'en écarter.

Les parties les plus volatiles, se vaporisant les premières, conduisent à un (NISH) supérieur à celui correspondant à la tension de vapeur du mélange. Par contre les échanges thermodynamiques entre les phases liquide et vapeur freinent la cavitation et retardent son developpement accentué.

Des abaques pratiques ont été publiés. Le critère B de Stepanoff a été introduit, mais donne lieu à discussion.

Les recherches se poursuivent.

Ici aussi l'écrasement des bulles est moins brutal qu'en eau froide.

\section{CHAPITRE IX}

\section{IX.1. Pompes supercaptivantes.}

Elles ont été introduites pour les applications où l'on doit tout subordonner au poids et à l'encombrement.
Elles utilisent des profils supercavitants, c'est-à-dire créant une poche de cavitation stable, ne se refermant qu'en aval de l'aube.

\section{CONCLUSION}

Le rapport constitue essentiellement un cadre destiné à faciliter la classification des renseignements et la poursuite des travaux du groupe.

\section{I S C U S I O N}

Président : M. BergenoN

M. le President remereie M. Guxox et son groupe de trayail pour l'efrort important que représente leur rapport. Il s'agit là d'un travail de base qui rassemble et classe les connaissances actuelles sur le sujet.

Il a en particulier pour but de metre a la disposition des turbiniers un document exposant la manière dont les constructeurs de pompes voient la cavitation, et lear expérience en la matière.

M. le Président souligne que M. Guiros a pris position en disant que, pour les roues de pompes en ánéral aucume formule en $\lambda$ n'avait permis de traduire l'allure d'ensemble des résultats obtenus, mais que, par contre, le graphique général de cavitation en fonction de $\varphi_{a}$ et $\psi_{a}$ permettait mieux de synthetiser les résultats.

M. le Puésident demande si quelqu'un veut prendre la parole car il $y$ a en quelques réactions parmi les assistants lorsque M. Gurton a dit qu'il arrivait que le bruit cesse completement quand la cavitation etait importante.

M. Cormaurt dit que ce fait s'explique fort bien, stant donné que lorsque la cavitation se développe, il y a formation d'un grand nombre de bulles de vapeur et de gaz au sein du liquide, ce qui a pour efret d'augmenter considérablement I'absorption acoustique du milieu. Le bruit est ainsi diminué jusqu'à devenir imperceptible.

M. DE Sant-Vaulry dit que le bruit ans une pompe est stirtout dû à ce que les bulles percutent sur la roue. Le bruit disparait si les bulles au lieu de percuter disparaissent dans le liquide lui-meme.

Le premier phenomene (pereussion) se produit lorsque la cavitation commence, e'est-à-dire lorsque la pression donnèe par la rone est voisine de la pression de marche sans cavitation.
Au contraire, le bruit disparaît lorsque la pression donnée par la roue en cavitation devient faible par rapport a la pression de marche. Cecl correspond a une cavitation profonde.

M. DE SAINT-VAUnx cite une expérience qu'il a faite avec une pompe multicollulaire a 5 roues. Louverture de la vame d'aspiration étant fixe, on ouvie de plus en plus la vanne de refoulement; la pression de refoulement baisse, depuis celle de marche normale jusqu’à 0 . On constate alors pour une faible cavitation un bruit intense. Pour une cavitation plus devée, le bruit disparaît. Le bruit leprend lorsque la pression n'est plus que les $4 / 5^{\text {es }}$ de celle d'origine. Ceci correspond au début de la cavitation de la 2 roue. Le bruit disparaît de nouveau, puis recommence sur la $3^{\circ}$ roue lorsque la pression diminue en dessous des $3 / 5^{\text {es }}$ de la pression d'origine.

Finalement, il est ainsi possible de compter le nombre de roues de la pompe en comptant le nombre de fois que le bruit s'intensifie pendant louverture de la vanne de refoulement.

M. le Président dit qu'une des principales préoccupations acfuelles est l'étude des machines, et en particulier des pompes, dans les zones de fonctionnement anormal (zone des gros débits au-dela du débit de rendement maximal; zone des petits débits au voisinage de la marche vanne fermée).

M. le Président remercie M. Gurrox et le groupe de travail de ce rapport, qu*il met a la disposition des constructeurs de turbines pour qu'ils examinent en particulier si la notion de vitesse specifique d'aspiration $S$ ne pourait pas leur être d'une utilité pratique.

M. le Président remercie, enfin, les persomes qui ont pris part à la discussion. 